\title{
OPEN Machine learning applied to near-infrared spectra for clinical pleural effusion classification
}

\author{
Zhongjian Chen ${ }^{1,2,3,4}$, Keke Chen ${ }^{1,2,3,4}$, Yan Lou ${ }^{5}$, Jing Zhu ${ }^{1,2,3}$, Weimin Mao ${ }^{1,2,3 凶}$ \& \\ Zhengbo Song ${ }^{1,2,3 凶}$
}

Lung cancer patients with malignant pleural effusions (MPE) have a particular poor prognosis. It is crucial to distinguish MPE from benign pleural effusion (BPE). The present study aims to develop a rapid, convenient and economical diagnostic method based on FTIR near-infrared spectroscopy (NIRS) combined with machine learning strategy for clinical pleural effusion classification. NIRS spectra were recorded for 47 MPE samples and 35 BPE samples. The sample data were randomly divided into train set $(n=62)$ and test set $(n=20)$. Partial least squares, random forest, support vector machine (SVM), and gradient boosting machine models were trained, and subsequent predictive performance were predicted on the test set. Besides the whole spectra used in modeling, selected features using SVM recursive feature elimination algorithm were also investigated in modeling. Among those models, NIRS combined with SVM showed the best predictive performance (accuracy: 1.0, kappa: 1.0 , and $\left.A U C_{R O C}: 1.0\right)$. SVM with the top 50 feature wavenumbers also displayed a high predictive performance (accuracy: 0.95 , kappa: $0.89, A \cup C_{R O C}: 0.99$ ). Our study revealed that the combination of NIRS and machine learning is an innovative, rapid, and convenient method for clinical pleural effusion classification, and worth further evaluation.

Pleural effusion is an abnormal accumulation of fluid in the pleural cavity. Two types of pleural effusions in clinics are: (1) transudative pleural effusion, resulting from fluid leaking into the pleural cavity, commonly caused by heart failure, cirrhosis, and post-surgery; (2) exudative pleural effusion, resulting from leaky blood vessels, mainly caused by cancer ${ }^{1,2}$, tuberculosis ${ }^{3}$, pulmonary embolism ${ }^{4}$, and pneumonia ${ }^{5}$. Exudative pleural effusion can be further classified as malignant or benign based on the detection of malignant cells in the pleural fluid. Pleural fluid of malignant pleural effusions (MPE) contains cancer cells, while benign pleural effusions (BPE) does not ${ }^{6}$. An accurate diagnosis of MPE is crucial, since MPE can be an indication of pleural metastases caused by lung cancer, breast cancer, ovarian cancer and lymphomas, with lung cancer being the leading cause ${ }^{7-9}$. Lung cancer patients with complicated MPE are usually classified as stage IV, and facing significantly different treatments and poor prognosis from those without $\mathrm{MPE}^{10}$. In the meantime, patients of pulmonary tuberculosis also show similar exudative effusions and overlapping symptoms as lung cancer, including short breath, chronic cough, fatigue and unexplained weight loss, making accurate diagnosis of MPEs more important and challenging8.

Current standard MPE diagnosis methods, including cytological and histological examinations, are not applicable for all cases. On the one hand, samples are hardly collected from malignant cells or tissues ${ }^{1}$. On the other hand, diagnoses for pulmonary tuberculosis usually need bacterial culture, which is time-consuming. Therefore, the undiagnosed effusion-especially when it is an undiagnosed MPE-may delay the treatment of lung cancer. Many cancer biomarkers, such as CEA, CA125, CA15-3, CA19-9, CYFRA21-1, and VEGF were investigated to help diagnose MPE. However, the sensitivity of the existing biomarkers was low: 54\% for CEA, 48\% for CA125, $51 \%$ for CA15-3, 25\% for CA19-9, 55\% for CYFRA21-1, 75\% for VEGF ${ }^{9,11-15}$. Therefore, an innovative diagnostic technique with a better sensitivity is needed.

Near-infrared spectroscopy (NIRS) is a spectroscopic tool using the near-infrared region of the electromagnetic spectrum from 780 to $2500 \mathrm{~nm}\left(4000 \mathrm{~cm}^{-1} \text { to } 12,820 \mathrm{~cm}^{-1}\right)^{16}$. NIRS has been utilized as a fast, non-invasive

\footnotetext{
${ }^{1}$ Cancer Hospital of the University of Chinese Academy of Sciences, Chinese Academy of Sciences, Banshandong Road\#1, Hangzhou 310000, Zhejiang Province, China. 'Zhejiang Cancer Hospital, Banshandong Road\#1, Hangzhou 310000, Zhejiang Province, China. ${ }^{3}$ Institute of Cancer and Basic Medicine (IBMC), Chinese Academy of Sciences, Hangzhou, China. ${ }^{4}$ College of Pharmaceutical Sciences, Zhejiang University, Yuhangtang Road\#866, Hangzhou 310000, Zhejiang Province, China. Intensive Care Unit, Zhejiang Medical \& Health Group Hangzhou Hospital, Banshan Kangjian Road \#1, Hangzhou 310000, Zhejiang Province, China. ${ }^{\square}$ email: maowm1218@163.com; zbszjch@163.com
} 


\begin{tabular}{|l|l|l|}
\hline Characteristics & MPE $(\mathbf{n}=\mathbf{4 7})$ & BPE $(\mathbf{n}=35)$ \\
\hline Age, years & $64 \pm 10$ & $49 \pm 19$ \\
\hline & \multicolumn{2}{|l|}{} \\
\hline Gender & $27(57.4 \%)$ & $26(74.3 \%)$ \\
\hline Male & $20(42.6 \%)$ & $9(25.7 \%)$ \\
\hline Female & Lung adenocarcinoma & Tuberculosis/Tuberculous pleurisy \\
\hline Cause &
\end{tabular}

Table 1. Demographic and clinical characteristics of malignant pleural effusion (MPE) and benign pleural effusion (BPE) cases.

tool for disease diagnosis, including cancer diagnosis, due to its ability of reflecting changes in molecular compositions by identifying different bonds vibrations in functional groups ${ }^{17-20}$. The variations in metabolites between MPE and BPE have been revealed by past metabonomic studies, indicated an increased amount of valine, lactate, alanine, lipids, and free fatty acids (FFAs) (16:0, 18:0, and 18:1) along with a decreased amount of acetoacetate, creatinine, $\beta$-glucose, and $\alpha$-glucose in $\mathrm{MPE}^{1,8,21}$. In addition, our previous metabolomics results revealed that the metabolites composition, such as lipids and oxidized polyunsaturated fatty acids, varies between MPE and BPE. Therefore, NIRS might be able to distinguish the differences between the chemical compositions of MPE and BPE, and contribute to a novel diagnosis method with a higher sensitivity.

In the present study, a total of 82 pleural effusion samples were analyzed, including $47 \mathrm{MPE}$ samples from diagnosed lung adenocarcinoma patients and 35 BPE samples from patients with diagnosed tuberculosis or tuberculous pleurisy. NIRS technology combined with machine learning approaches, including partial least squares (PLS), random forest (RF), support vector machine (SVM), and gradient boosting machine (GBM) models, were used to screen for the characteristics in near-infrared spectra between MPE and BPE samples.

\section{Materials and methods}

Pleural effusion samples. A total of 82 pleural effusion samples were obtained from the biobank of Zhejiang Cancer Hospital in Hangzhou, China. MPE samples were collected from 47 patients diagnosed with lung adenocarcinoma, complicated with pleural metastases. BPE samples were collected from 35 patients diagnosed with pulmonary tuberculosis and/or tuberculous pleurisy. Informed consent was obtained from all individual participants included in the study, and our study was approved by the Ethics Committee of Zhejiang Cancer Hospital. All methods were performed in accordance with the relevant guidelines and regulations. The diagnoses were based on cytological or histological examinations for MPE and bacterial culture which were performed in the cases of tuberculosis. All the pleural effusion samples were spun at $1600 \mathrm{~g}$ for $10 \mathrm{~min}$ at $4{ }^{\circ} \mathrm{C}$, and the aliquot of supernatant was stored at $-80^{\circ} \mathrm{C}$ until analysis. Basic information of patients, including gender, age, and pathological information, were collected (Table 1).

NIRS analysis and spectra collection. The frozen samples were thawed to room temperature before analysis. The NIR spectra of the pleural effusions were collected using an Antaris ${ }^{\text {Th }}$ II FT-NIR analyzer (Thermo Nicolet, USA) with air as a reference. Aquartz colorimetric tube with an optical path of $2 \mathrm{~mm}$ was used as the sample cup. Each spectrum was obtained from 32 successive scans from 4000 to $10,000 \mathrm{~cm}^{-1}$ with a spectral resolution of $4 \mathrm{~cm}^{-1}$. The spectrum was recorded by absorbance. Each sample was analyzed in triplicate and the average spectrum was calculated by TQ Analyst 8.0 data processing software.

Data analysis. Randomly slicing the data into train set and test set and preprocess. This cohort was randomly split into a train set of 62 cases (36 MPE and $26 \mathrm{BPE}$ ) and test set of 20 cases (11 MPE and $9 \mathrm{BPE}$ ) using "sample" function in R.

Preprocess. Spectra data in train set were mean-centered, scaled to unit variance, smoothed using a SavitzkyGolay filter, and dimensionally reduced by PCA analysis via generation of audit data summarizing discrete variables. Spectra in test set were preprocessed with the same method and the same values of parameters in train set.

Model training and testing. For PLS, RF, and GBM, model training and parameter tuning were conducted with caret $\mathrm{R}$ package, in which 10 repeated, fivefold cross validation was used. For SVM, model training was performed using e1071 R package with fivefold cross validation. Accuracy was used to select the optimal model by the largest value. The running time for each model was measured in the following CPU condition: Intel(R) Core (TM) i5-8250U CPU@ 1.60 GHz.

Feature wavelength selection with SVM-RFE algorithm. SVM-RFE algorithm was used to rank the wavenumbers in train set. The algorithm processes were briefly described as follows: (1) train the SVM model; (2) compute the weight vector; (3) rank the variables from the minimum to the maximum by square weights; (4) update the feature ranking list; (5) eliminate the feature with the smallest square weight, and repeat from Step 1 until all the 
A

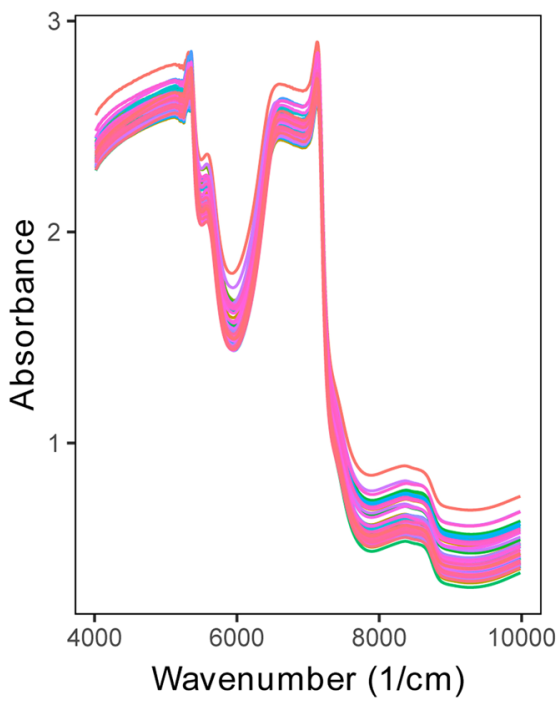

B

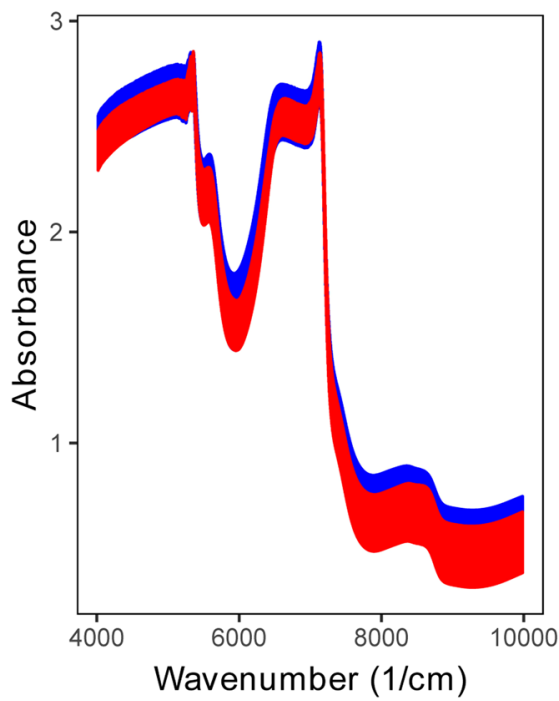

C

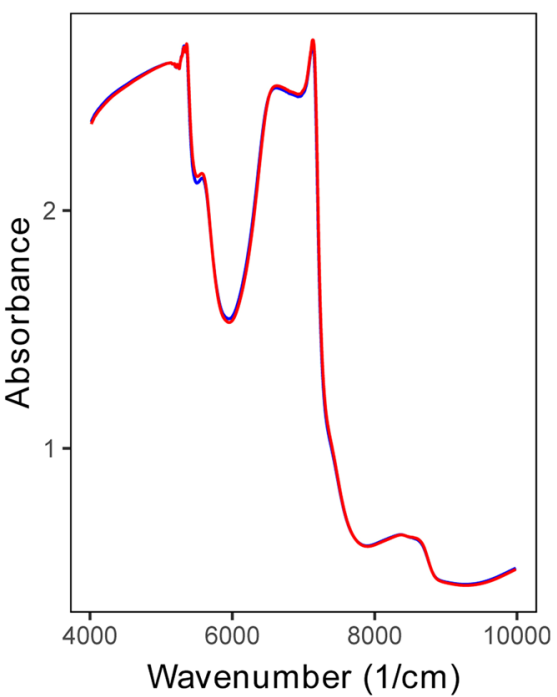

Figure 1. Raw NIR spectra of 82 pleural effusion samples (A), color-label MPE (red) and BPE (blue) NIR spectra (B), and the average NIR spectra for MPE (red) and BPE (blue) samples.

features were ranked. In order to optimize the subset size of the features, a series of subsets with different sizes of wavenumber (from top 1 to the total number) were evaluated for their predictive performances.

\section{Results}

NIR spectral analysis. Plots of the raw NIR spectra of the 82 pleural effusions, groups of MPE and BPE samples, and their average spectra were illustrated in Fig. 1. Evidently, due to the broad and overlapping spectra peak, there was no significant difference between MPE and BPE samples in raw spectra, and the direct interpretation is nearly impossible. However, though there were no feature peaks, the NIR spectra still contain a lot of information in terms of the chemical composition of pleural effusion. There are four regions referring to different chemical substructures: wavenumbers between 4200 and $5500 \mathrm{~cm}^{-1}$ indicate the $\mathrm{CH}, \mathrm{OH}$ and NH stretch/ $\mathrm{CH}$ deformations in the phenyl; between 5400 and $6100 \mathrm{~cm}^{-1}$ refers to the first overtone of $\mathrm{CH}$; wavenumbers of 6200 to $7600 \mathrm{~cm}^{-1}$ indicate the first overtone of $\mathrm{OH}, \mathrm{NH}$, and $\mathrm{CH}$; and wavelengths of 7900 to $9000 \mathrm{~cm}^{-1}$ indicate the second overtone of $\mathrm{CH}$. NH, and $\mathrm{CH}$ combinations were denoted by wavenumbers of 6200 to $7600 \mathrm{~cm}^{-1}$; and the second overtone of $\mathrm{CH}$ was denoted by wavelengths of 7900 to $9000 \mathrm{~cm}^{-117,22}$.

Principal component analysis. As an unsupervised model, a principal component analysis (PCA) was performed to check the extent of clustering of the samples and to investigate the potential NIR features for differentiating between MPE and BPE classes. Figure 2 shows a scatter plot of the first two principal components (PCs), accounting for about $94.7 \%$ of the total variation. However, there was no clear separation between MPE and BPE samples, which indicated that the structure or the relationship of the data might be complicated, nonlinear, and therefore unfit for an unsupervised model.

Predictive performances in pleural effusion classification. For PLS model, the optimized number of components used in model was 3 , and the time for running the model is $1.05 \mathrm{~s}$. The predictive accuracy, kappa, and $\mathrm{AUC}_{\mathrm{ROC}}$ in the test set were $0.91,0.67$, and 0.94 , respectively (Figs. 3A,B, 4A; Table 2).

For RF model, the optimized value for mtry is 32 , and the time used for running the model is $8.25 \mathrm{~s}$. The predictive accuracy, kappa, and $\mathrm{AUC}_{\mathrm{ROC}}$ in the test set were 0.95 and $0.90,1.00$, respectively (Figs. 3C,D, 4B; Table 2).

SVM model performs best with "linear" kernel, cost value of 5 and number of support vectors of 19, and the running time is $0.52 \mathrm{~s}$. The predictive accuracy, kappa, and $\mathrm{AUC}_{\mathrm{ROC}}$ in test were $1.00,1.00$, and 1.00, respectively (Figs. 3E,F, 4C; Table 2).

For GBM model, the final optimized model was with the following parameters: ntrees value of 50, interaction depth value of 1 , shrinkage value of 0.1 and $n$ minobsinnode value of 10 . The running time was $5.57 \mathrm{~s}$. The predictive accuracy, kappa, and $\mathrm{AUC}_{\mathrm{ROC}}$ were $0.95,0.9$, and 0.99 , respectively (Figs. 3G,H, 4D; Table 2).

Among the four models, the performance of PLS was unsatisfactory. RF and GBM have exhibited relatively high accuracy and kappa values in both train and test sets, but with a relatively longer computational time. In contrast, SVM was the fastest model in computation and has displayed the best predictive performance in the test group. Therefore, SVM was considered as the best model for pleural effusion classification in this study. More detailed model performance parameters were illustrated in Table 2.

Wavenumber selection. After ranking wavenumbers by SVM-RFE algorithm, SVM model with different sizes of featured wavenumbers was tested (from the top 1 to all the features). The results showed that the predic- 


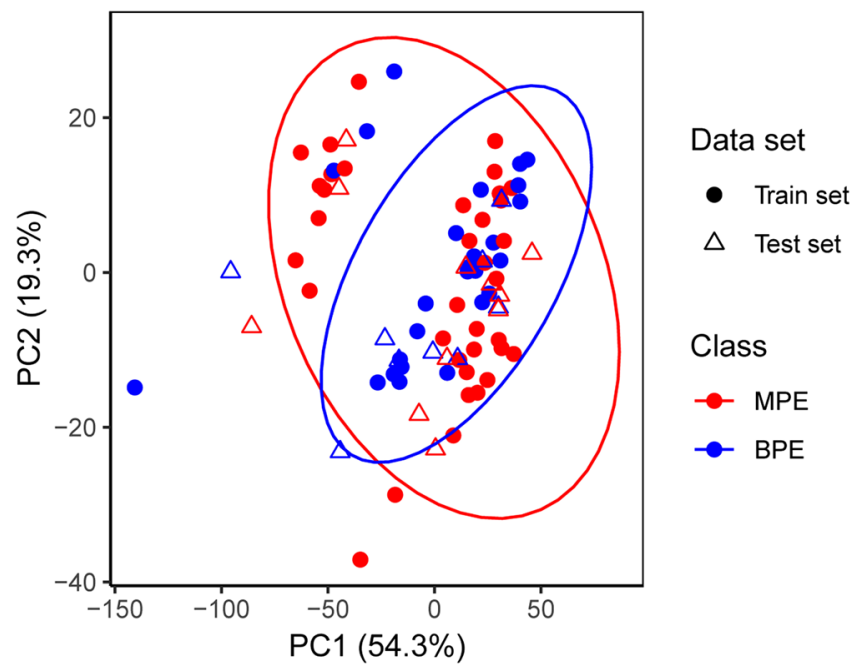

Figure 2. Scatter plots of the first two principal components (PCs). The variance explained PC1 and PC2 were $54.3 \%$ and $19.3 \%$, respectively. 82 cases were randomly split into train set $(n=62$, filled circle) and test set $(\mathrm{n}=20$, hollow triangle). 47 MPE cases are colored red, and 35 BPE cases are colored blue.

A

PLS Training

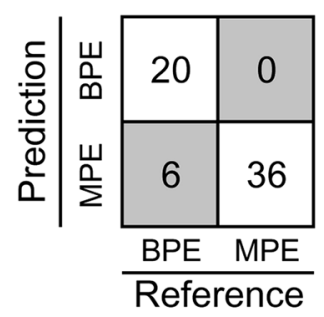

E

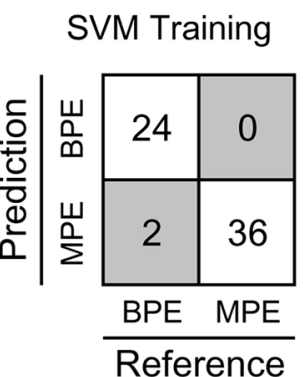

B

PLS Testing

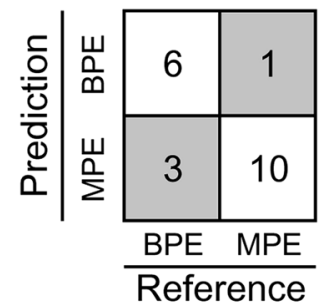

F

SVM Testing

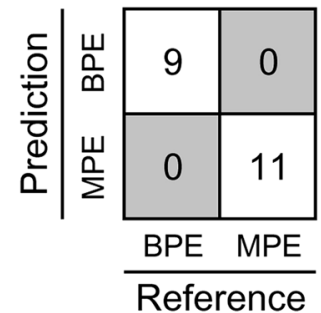

C

RF Training

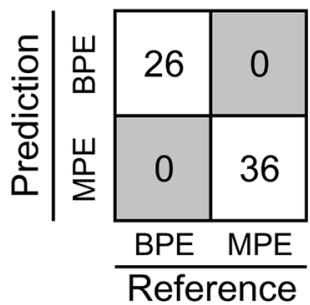

G

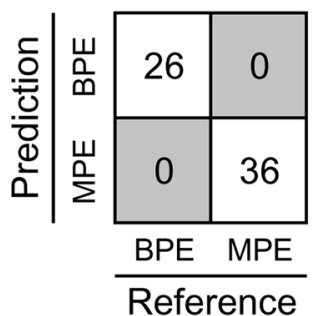

$\mathrm{H}$

GBM Testing

RF Testing
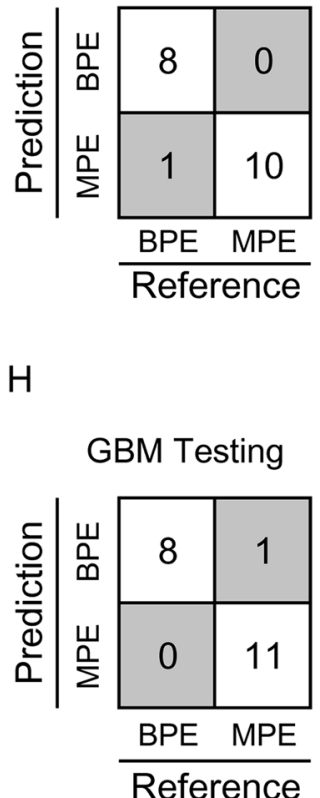

Figure 3. Predictive performance of the 4 models in train set and test set. PLS modeling in train set (A) and test set (B); RF modeling in train set (C) and test set (D); SVM modeling in train set (E) and test set (F); GBM modeling in train set $(\mathbf{G})$ and test set $(\mathbf{H})$. Good prediction is defined when predictive class is the same as the reference (no background), otherwise bad prediction is defined (gray background).

tive accuracy increased dramatically, exceeding 0.90 (0.79 kappa) in both train and test sets within the size of 4 , and then slowly reached 0.95 (0.89 kappa) at the size of 46 (Fig. 5A,B). The highest predictive accuracy reached 0.97 (0.93 kappa) (at the size of 160) in the train set, and 1.00 (1.00 kappa) (at the size of 102) in the test set. Finally, the top 50 featured wavenumbers were selected as variables in SVM model. Figure 5C displayed the distribution of the top $4\left(6626 \mathrm{~cm}^{-1}, 5311 \mathrm{~cm}^{-1}, 6622 \mathrm{~cm}^{-1}\right.$, and $\left.6309 \mathrm{~cm}^{-1}\right)$ and the top 50 featured wavenumbers. SVM model with the top 50 features had an $\mathrm{AUC}_{\mathrm{ROC}}$ of 0.99 for predicting the pleural effusion classes (Fig. 5D). 
A

PLS

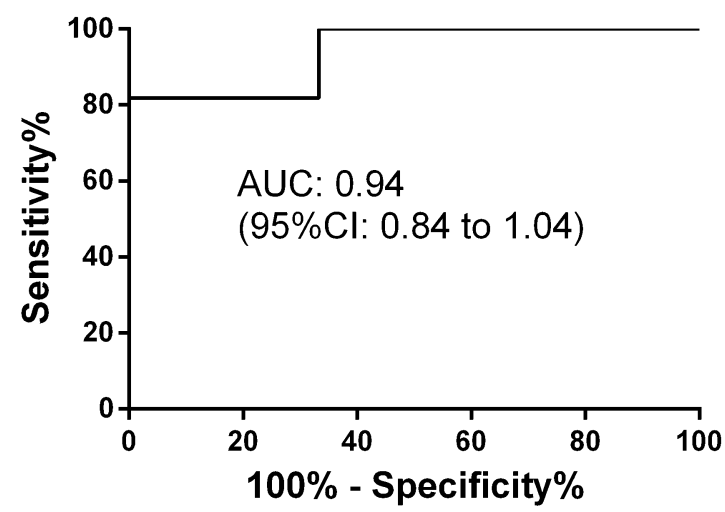

C

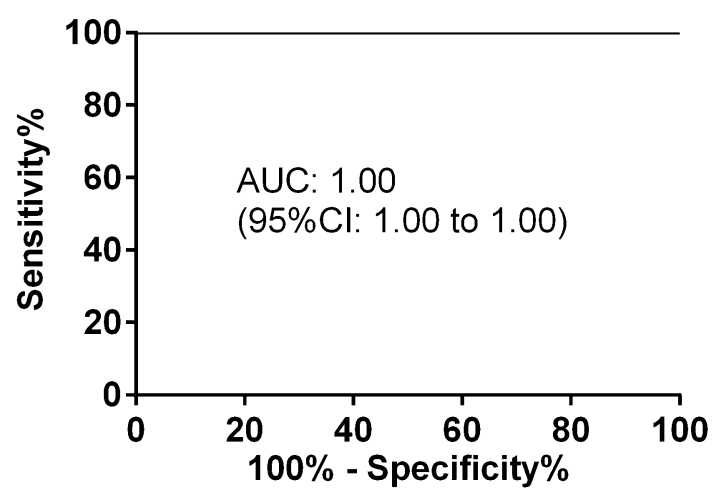

B

RF

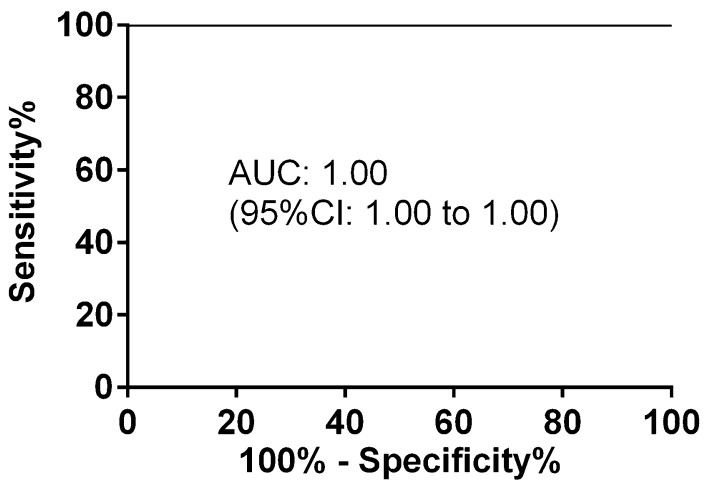

D

GBM

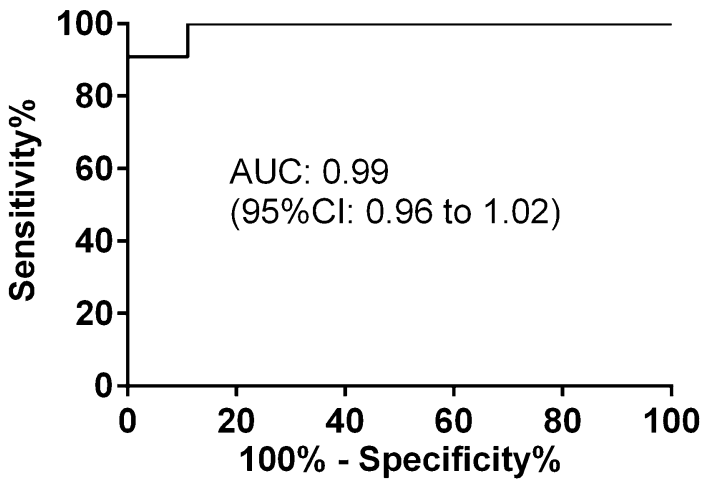

Figure 4. Receiver operating curve (ROC) in predicting pleural effusion classes for the test set. (A) Result from PLS model; (B) result from RF model; (C) result from SVM model; (D) result from GBM model.

\begin{tabular}{|l|l|l|l|l|l|}
\hline Data set & Performance & PLS & RF $^{\mathbf{c}}$ & SVM $^{\mathbf{d}}$ & GBM $^{\mathbf{e}}$ \\
\hline \multirow{5}{*}{ Train set $(\mathrm{n}=62)$} & Accuracy & 0.90 & 1.00 & 0.96 & 1.00 \\
\cline { 2 - 6 } & Kappa & 0.79 & 1.00 & 0.93 & 1.00 \\
\cline { 2 - 6 } & Sensitivity & 1.00 & 1.00 & 1.00 & 1.00 \\
\cline { 2 - 6 } Test set $(\mathrm{n}=20)$ & Specificity & 0.77 & 1.00 & 0.92 & 1.00 \\
\hline & Accuracy & 0.80 & 0.95 & 1.00 & 0.95 \\
\cline { 2 - 6 } & Kappa & 0.59 & 0.90 & 1.00 & 0.90 \\
\cline { 2 - 6 } & Sensitivity & 0.91 & 1.00 & 1.00 & 0.91 \\
\cline { 2 - 6 } & Specificity & 0.67 & 0.89 & 1.00 & 1.00 \\
\hline
\end{tabular}

Table 2. Predictive performance of the 4 machine learning approaches in training and testing sets in the present study. ${ }^{a}$ Predictive performance was calculated using confusionMatrix function from caret package in R. ${ }^{\mathrm{b}}$ Partial least squares, PLS. ${ }^{\mathrm{c}}$ Random forest, RF. ${ }^{\mathrm{d}}$ Support vector machines, SVM. ${ }^{\mathrm{e}}$ Gradient boosting machine, GBM; PLS, RF, and GBM modeling were performed using caret $\mathrm{R}$ package, and SVM modeling was conducted using e1071 R package.

\section{Discussion and conclusion}

Our study applied several machining learning approaches in NIRS analysis to classify malignant and benign pleural effusion samples, through which a rapid, convenient and accurate diagnostic method was successfully developed.

The diagnostic performance of NIRS has been investigated in the past studies. For example, Chen et al. established a NIRS based method to distinguish between normal and malignant colorectal tissues ${ }^{17}$. However, to the best of our knowledge, our study is the first one that applyed NIRS to the classification of pleural effusion. MPE usually indicates advanced development in cancer, which contributes to a unique cancerous microenvironment that is significantly different from the surrounding healthy tissues, featured with variations in metabolites including proteins and lipids ${ }^{1,8,10,21}$. Therefore, NIRS can be used to distinguish the variation of chemicals in samples ${ }^{17-20}$. 
A

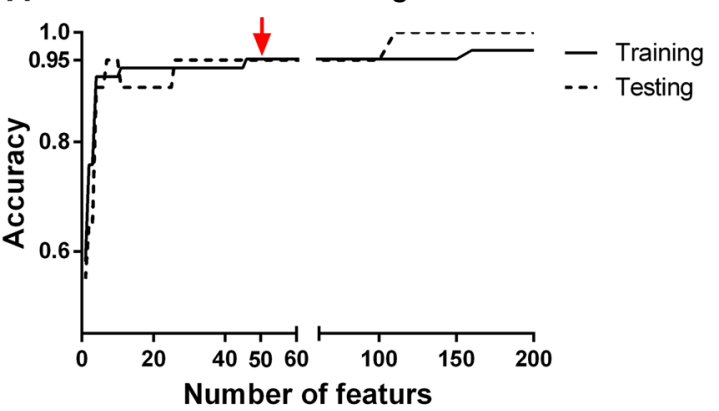

C

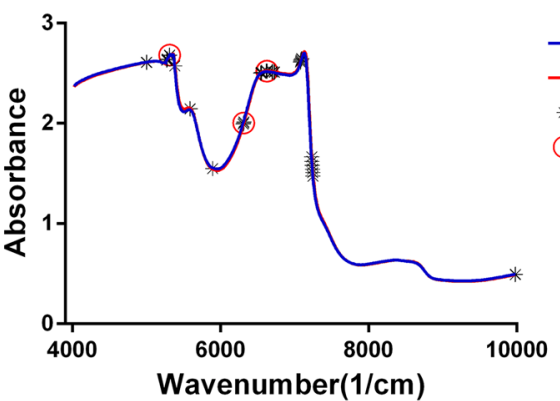

B

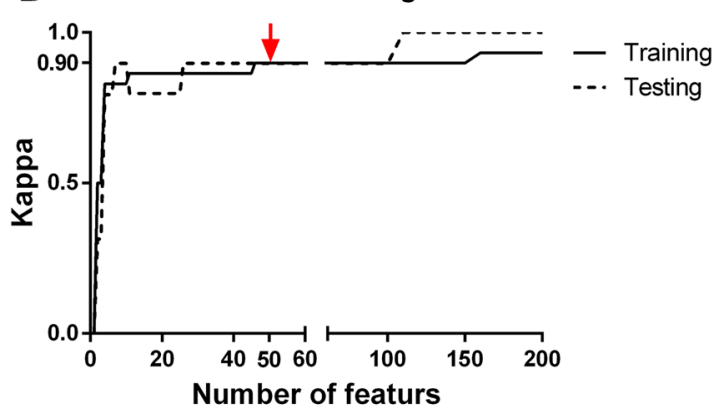

D

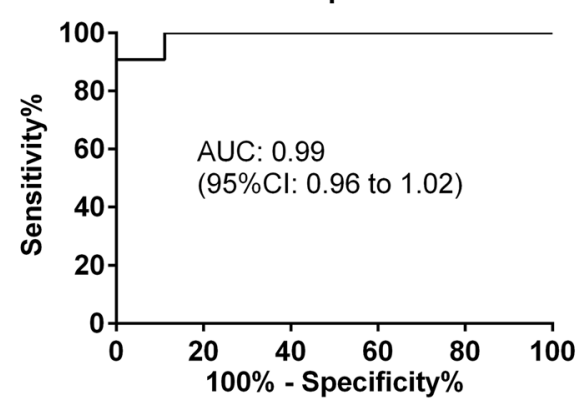

Figure 5. SVM modeling with different sizes of feature wavenumbers. The curves of predictive accuracy (A) and kappa (B) values increase as the sizes of top features grow. Top 50 feature wavenumbers are selected in final SVM, and the top 50 feature wavenumbers (including top 4) were marked on the average spectra; (D) ROC in predicting pleural effusion classes for the test set by SVM mode with top 50 features.

Although the NIRS of malignant and benign samples overlapped to a great extent, additional application of machine learning aided in the separation of malignant and benign samples and some spectral regions that are of high diagnostic values were detected. According to our previous metabolomics results using the same samples, malignant pleural effusion differs from benign samples in metabolites like acylcarnitines, oxidized polyunsaturated fatty acids (PUFAs), and ether lipids ${ }^{23}$. In line, the top 50 diagnostic wavenumbers detected by SVM-RFE denoted functional groups including $\mathrm{CH}, \mathrm{CH} 2$, and $\mathrm{CH} 3, \mathrm{NH}$, free and bound $\mathrm{OH}$. The spectral intervals of $\mathrm{CH} 2$ and $\mathrm{CH} 3$ arisen from stretching vibrations at 5577 to $5889 \mathrm{~cm}^{-1}$ of the first overtone, and that of $\mathrm{OH}$ of stretching vibrations at 7077 to $7093 \mathrm{~cm}^{-1}$ and at 9977 to $9981 \mathrm{~cm}^{-1}$ denoted the change in ether lipids. In addition, $\mathrm{CH}$ group of combined vibrations of second overtone was detected at 7227 to $7247 \mathrm{~cm}^{-1}$ together with the aforementioned $\mathrm{OH}$ groups explained the existence of oxidized PUFAs. Acylcarnitines can also be annotated in terms of the $\mathrm{NH}$ (at 6306 to $6618 \mathrm{~cm}^{-1}$ ), $\mathrm{CH}$, and $\mathrm{OH}$ detected.

Compared to the traditional diagnostic methods, such as cytological or histological examinations, our method is simpler and more convenient since the supernatant of the pleural effusion sample is the only need. Hence our method could be a supplementary tool when there are difficulties in collecting malignant cells or tissues. In addition, compared to the high throughput method, such as metabolomics, our NIRS method is more economical, less time- and labor-consuming, and needs no additional sample preparation. Altogether, our NIRS method is worthy to be further developed for clinical application.

At present, NIRS-SVM has been considered as the best model for pleural effusion classification. SVM algorithm is fast and has a high predictive performance for pleural effusion classification. Compared to models using the whole spectra, SVM with the top 50 features is less complex and more stable in application, and worthy to be further investigated.

The major limitation in our study is that our cohort size was relatively small. In addition, the types of pleural effusions were too limited. A larger cohort with more types of pleural effusions could be studied in the future. In conclusion, our study provided an idea that NIRS could be a helpful tool in the classification of pleural effusion, with advantages of high speed and accuracy, which might improve the current clinic diagnostic methods for MPE.

Received: 20 August 2020; Accepted: 31 March 2021

Published online: 03 May 2021

\section{References}

1. Zhou, X. M. et al. Metabonomic classification and detection of small molecule biomarkers of malignant pleural effusions. Anal. Bioanal. Chem. 404, 3123-3133. https://doi.org/10.1007/s00216-012-6432-6 (2012).

2. Lim, J. U. et al. Elevated pretreatment platelet-to-lymphocyte ratio is associated with poor survival in stage IV non-small cell lung cancer with malignant pleural effusion. Sci. Rep. 9, 4721. https://doi.org/10.1038/s41598-019-41289-9 (2019). 
3. Shi, J. et al. Potential biomarkers for antidiastole of tuberculous and malignant pleural effusion by proteome analysis. Biomark. Med. 13, 123-133. https://doi.org/10.2217/bmm-2018-0200 (2019).

4. Olgun Yildizeli, S. et al. Pleural effusion as an indicator of short term mortality in acute pulmonary embolism. Tuberkuloz ve toraks 66, 185-196. https://doi.org/10.5578/tt.67203 (2018).

5. Bai, K. J. et al. Alterations by air pollution in inflammation and metals in pleural effusion of pneumonia patients. Int. J. Environ. Res. Public Health 16, 3. https://doi.org/10.3390/ijerph16050705 (2019).

6. Walker, S., Bibby, A. C. \& Maskell, N. A. Current best practice in the evaluation and management of malignant pleural effusions. Ther. Adv. Respir. Dis. 11, 105-114. https://doi.org/10.1177/1753465816671697 (2017).

7. Roncella, S. et al. Diagnosis and origin determination of malignant pleural effusions through the use of the breast cancer marker human mammaglobin. Diagn. Mol. Pathol. Am. J. Surg. Pathol. Part B 19, 92-98. https://doi.org/10.1097/PDM.0b013e3181ba6c78 (2010).

8. Wang, C., Peng, J., Kuang, Y., Zhang, J. \& Dai, L. Metabolomic analysis based on 1H-nuclear magnetic resonance spectroscopy metabolic profiles in tuberculous, malignant and transudative pleural effusion. Mol. Med. Rep. 16, 1147-1156. https://doi.org/10. 3892/mmr.2017.6758 (2017).

9. Chen, Y., Mathy, N. W. \& Lu, H. The role of VEGF in the diagnosis and treatment of malignant pleural effusion in patients with nonsmall cell lung cancer (review). Mol. Med. Rep. 17, 8019-8030. https://doi.org/10.3892/mmr.2018.8922 (2018).

10. Murthy, P. et al. Making cold malignant pleural effusions hot: driving novel immunotherapies. Oncoimmunology 8, e1554969. https://doi.org/10.1080/2162402X.2018.1554969 (2019).

11. Nam, H. S. Malignant pleural effusion: medical approaches for diagnosis and management. Tuberc. Respir. Dis. 76, 211-217. https:// doi.org/10.4046/trd.2014.76.5.211 (2014).

12. Lai, R. S., Chen, C. C., Lee, P. C. \& Lu, J. Y. Evaluation of cytokeratin 19 fragment (CYFRA 21-1) as a tumor marker in malignant pleural effusion. Jpn. J. Clin. Oncol. 29, 421-424 (1999).

13. Neskovic-Konstantinovic, Z. et al. CEA and CA 15-3 in pleural effusion of advanced breast cancer patients: clinical relevance and diagnostic value. Int. J. Biol. Mark. 8, 94-102 (1993).

14. Jibiki, K., Demura, R., Abe, Y., Odagiri, E. \& Demura, H. A clinical evaluation of CA 125 levels in serum, ascites and pleural effusion. Jpn. J. Cancer Clin. 33, 1756-1762 (1987).

15. Wu, G. P., Ba, J., Zhao, Y. J. \& Wang, E. H. Diagnostic value of CEA, CYFRA 21-1, NSE and CA 125 assay in serum and pleural effusion of patients with lung cancer. Acta Cytol. 51, 679-680 (2007).

16. Xiaobo, Z., Jiewen, Z., Povey, M. J., Holmes, M. \& Hanpin, M. Variables selection methods in near-infrared spectroscopy. Anal. Chim. Acta 667, 14-32. https://doi.org/10.1016/j.aca.2010.03.048 (2010).

17. Chen, H., Lin, Z., Mo, L., Wu, T. \& Tan, C. Near-infrared spectroscopy as a diagnostic tool for distinguishing between normal and malignant colorectal tissues. Biomed. Res. Int. 2015, 472197. https://doi.org/10.1155/2015/472197 (2015).

18. Cheng, P. et al. Near-infrared fluorescence probes to detect reactive oxygen species for keloid diagnosis. Chem. Sci. 9, 6340-6347. https://doi.org/10.1039/c8sc01865k (2018).

19. Koike, S. et al. Application of functional near infrared spectroscopy as supplementary examination for diagnosis of clinical stages of psychosis spectrum. Psychiatry Clin. Neurosci. 71, 794-806. https://doi.org/10.1111/pcn.12551 (2017).

20. Rotgans, J. I. et al. Evidence supporting dual-process theory of medical diagnosis: a functional near-infrared spectroscopy study. Med. Educ. 53, 143-152. https://doi.org/10.1111/medu.13681 (2019).

21. Lam, C. W. \& Law, C. Y. Untargeted mass spectrometry-based metabolomic profiling of pleural effusions: fatty acids as novel cancer biomarkers for malignant pleural effusions. J. Proteome Res. 13, 4040-4046. https://doi.org/10.1021/pr5003774 (2014).

22. Li, Y. et al. An approach combining real-time release testing with near-infrared spectroscopy to improve quality control efficiency of Rhizoma paridis. Spectrochim. Acta Part A Mol. Biomol. Spectrosc. 157, 186-191. https://doi.org/10.1016/j.saa.2016.01.001 (2016).

23. Yang, Z. et al. Metabolic and lipidomic characterization of malignant pleural effusion in human lung cancer. J. Pharm. Biomed. Anal. 180, 113069. https://doi.org/10.1016/j.jpba.2019.113069 (2020).

\section{Acknowledgements}

The authors would like to thank Professor Yong jiang Wu and his students, Siyu Zhang and Yang Chen, from the College of Pharmaceutical Science at Zhejiang University for providing their expertise in NIRS analysis. This work was supported by the National Natural Science Foundation of China (Grant Numbers 81672315, 81802276, and 81302840) and Key R\&D Program Projects in Zhejiang Province (Grant Number 2017C04G1360498).

\section{Author contributions}

W.M., Z.S.: Conceptualization, project administration, data curation, supervision; Z.C.: Formal analysis, methodology; Y.L., J.Z.: Validation; Z.C., K.C.: Writing-original draft; Z.C., K.C.: Writing—review and editing.

\section{Competing interests}

The authors declare no competing interests.

\section{Additional information}

Supplementary Information The online version contains supplementary material available at https://doi.org/ 10.1038/s41598-021-87736-4.

Correspondence and requests for materials should be addressed to W.M. or Z.S.

Reprints and permissions information is available at www.nature.com/reprints.

Publisher's note Springer Nature remains neutral with regard to jurisdictional claims in published maps and institutional affiliations. 
(c) (i) Open Access This article is licensed under a Creative Commons Attribution 4.0 International cc) License, which permits use, sharing, adaptation, distribution and reproduction in any medium or format, as long as you give appropriate credit to the original author(s) and the source, provide a link to the Creative Commons licence, and indicate if changes were made. The images or other third party material in this article are included in the article's Creative Commons licence, unless indicated otherwise in a credit line to the material. If material is not included in the article's Creative Commons licence and your intended use is not permitted by statutory regulation or exceeds the permitted use, you will need to obtain permission directly from the copyright holder. To view a copy of this licence, visit http://creativecommons.org/licenses/by/4.0/.

(C) The Author(s) 2021 